\title{
Implantation of Liquid Nitrogen Frozen Tumor Tissue after Posterior Decompression and Stabilization for Metastatic Spinal Tumors
}

\author{
Kazuya Shinmura, Hideki Murakami, Satoru Demura, Satoshi Kato, Katsuhito Yoshioka, Hiroyuki Hayashi, \\ Noriaki Yokogawa, Takashi Igarashi, Moriyuki Fujii, Noritaka Yonezawa, Hiroyuki Tsuchiya \\ Department of Orthopaedic Surgery, Graduate School of Medical Sciences, Kanazawa University, Kanazawa, Japan
}

\begin{abstract}
Study Design: A retrospective study.
Purpose: To evaluate the immunity-enhancing effect of implantation of a liquid nitrogen-treated tumor.

Overview of Literature: We have developed a new technique of implanting a tumor frozen in liquid nitrogen after posterior decompression and stabilization, with the aim of enhancing antitumor immunity in order to prolong the survival period of the patient. In the current study, the immunity-enhancing effect of this new technique has been evaluated.

Methods: The subjects were 19 patients in whom we had earlier performed decompression and stabilization between April 2011 and September 2013. The 19 subjects were divided into two groups, namely a frozen autologous tumor tissue implantation group ( $n=15$; "implantation group"), which consisted of patients, who underwent implantation with autologous tumor tissue frozen in liquid nitrogen, and a control group $(n=4)$, which consisted of patients, who did not undergo autologous cancer transplantation. To evaluate the immunity-enhancing effect of the protocol, plasma cytokines (interferon [IFN]- $\gamma$ and interleukin [IL]-12) were analyzed before surgery and a month after surgery.

Results: The mean rate of increase in IFN- $\gamma$ was significantly higher in the implantation group ( $p=0.03)$. Regarding IL-12, no significant difference was observed between the groups, although the implantation group exhibited increased levels of IL-12 ( $p=0.22$ ).

Conclusions: Decompression and stabilization combined with autologous frozen tumor cell implantation can enhance cancer immunity in metastatic spinal tumor patients. It is hypothesized that this procedure might prevent local recurrence and prolong survival period.
\end{abstract}

Keywords: Metastatic spinal tumor; Liquid nitrogen; Posterior decompression and stabilization; Antitumor immunity

\section{Introduction}

The purposes of posterior decompression and stabilization for metastatic spinal tumors are to maintain quality of life of the patients and improve paralysis. In this regard, the effectiveness of the procedure has been demonstrated in various studies [1].

Previously, we have performed posterior decompression and stabilization in patients with metastatic spinal tumors presenting with progressive paralysis, with an estimated prognosis of at least more than 3 months. In such cases, posterior decompression and stabilization was performed

\footnotetext{
Received Apr 06, 2015; Accepted Apr 28, 2015

Corresponding author: Kazuya Shinmura

Department of Orthopaedic Surgery, Graduate School of Medical Sciences, Kanazawa University,

13-1 Takara-machi, Kanazawa, 920-8641, Japan

Tel: +81-76-265-2374, Fax: +81-76-234-4261,,E-mail: kazuyashinmura@yahoo.co.jp
} 
at the earliest and to the extent permitted by the general condition of the patient. However, the procedure is not curative but palliative and therefore cannot prolong prognosis. In addition, local recurrence is an unignorable concern in patients, who demonstrate long-term survival. Furthermore, surgical stress has been indicated to possibly exacerbate metastasis by attenuating cell-mediated immunity, which plays an important role against cancer [2]. Since April 2011, we have combined decompression and stabilization with autologous tumor tissue implantation, in which a part of the resected tumor is frozen by liquid nitrogen and implanted subcutaneously (Fig. 1). This procedure is anticipated to prevent local recurrence and prolong the survival period by enhancement of anitcancer immunity. With this therapy, anti-tumor immunity may be enhanced by the release of tumor antigens from the frozen, dead tumor cells via dendritic cells. In the present study, the anti-tumor immunity enhancement effect of the operative procedure was examined.

\section{Materials and Methods}

\section{Ethics statements}

This study was approved by the ethics committee of Kanazawa (Japan) University. Written informed consent for the surgery and entry into the research study was obtained from all 19 patients.

\section{Patients characteristics}

The subjects were 19 patients, in whom we performed decompression and stabilization between April 2011 and September 2013; these patients had metastatic spinal tumors that resulted in acute neurological disorders. The subjects were divided into two groups, namely a frozen autologous tumor tissue implantation group $(n=15$; hereafter referred to as "implantation group"), which consisted of patients, who underwent implantation with autologous tumor tissue frozen with liquid nitrogen, and a control group $(n=4)$, which consisted of patients, who did not undergo autologous cancer transplantation. The implantation group comprised 7 men and 8 women, with a mean age of 56.3 years (range, 38-74 years). The tumor site was the cervical spine in one case, the thoracic spine in 11 cases, and the lumbar spine in 3 cases. The primary lesions were breast cancer in 5 cases; prostate, renal, and rectal cancers in 2 cases each; and lung, pancreas, cervical, and laryngeal cancers in one case each. The control group comprised of only 4 men, with a mean age of 62.2 years (range, 55-68 years). The tumor site was the thoracic spine in 3 cases and the lumbar spine in one case. The primary lesions were histiocytic sarcoma in 2 cases, prostate cancer in one case, and parotid cancer in another case.

\section{Surgical procedure}

1) Posterior decompression and stabilization

Decompression and stabilization was performed using a posterior approach in all the cases. The patients were operated using the posterior approach as follows. The tumor mass and lamina of the afflicted vertebral body were resected, and the spinal cord was decompressed. Pedicle screws were inserted on both sides of the two vertebral bodies above and below the afflicted vertebral body and fixated with two rods (one on the left and one on the right). However, bone grafting was not performed.

2) Implantation of frozen autologous tumor tissue Implantation was performed only in the case of implanta-

\section{Liquid nitrogen $\left(-196^{\circ} \mathrm{C}\right)$}
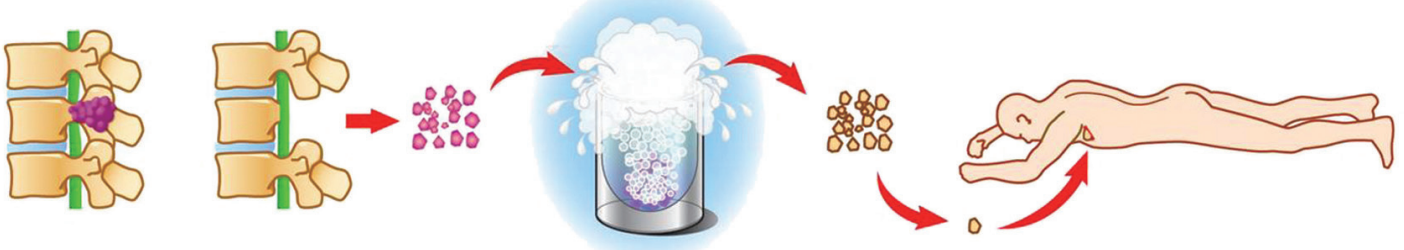

20 min.

Fig. 1. Implantation of frozen tumor tissue treated with liquid nitrogen. The tumor tissue was immersed in liquid nitrogen $\left(-196^{\circ} \mathrm{C}\right)$ for 20 minutes. Subsequently, the liquid nitrogen-treated tumor tissue was implanted into the subcutaneous tissue of the axilla. 

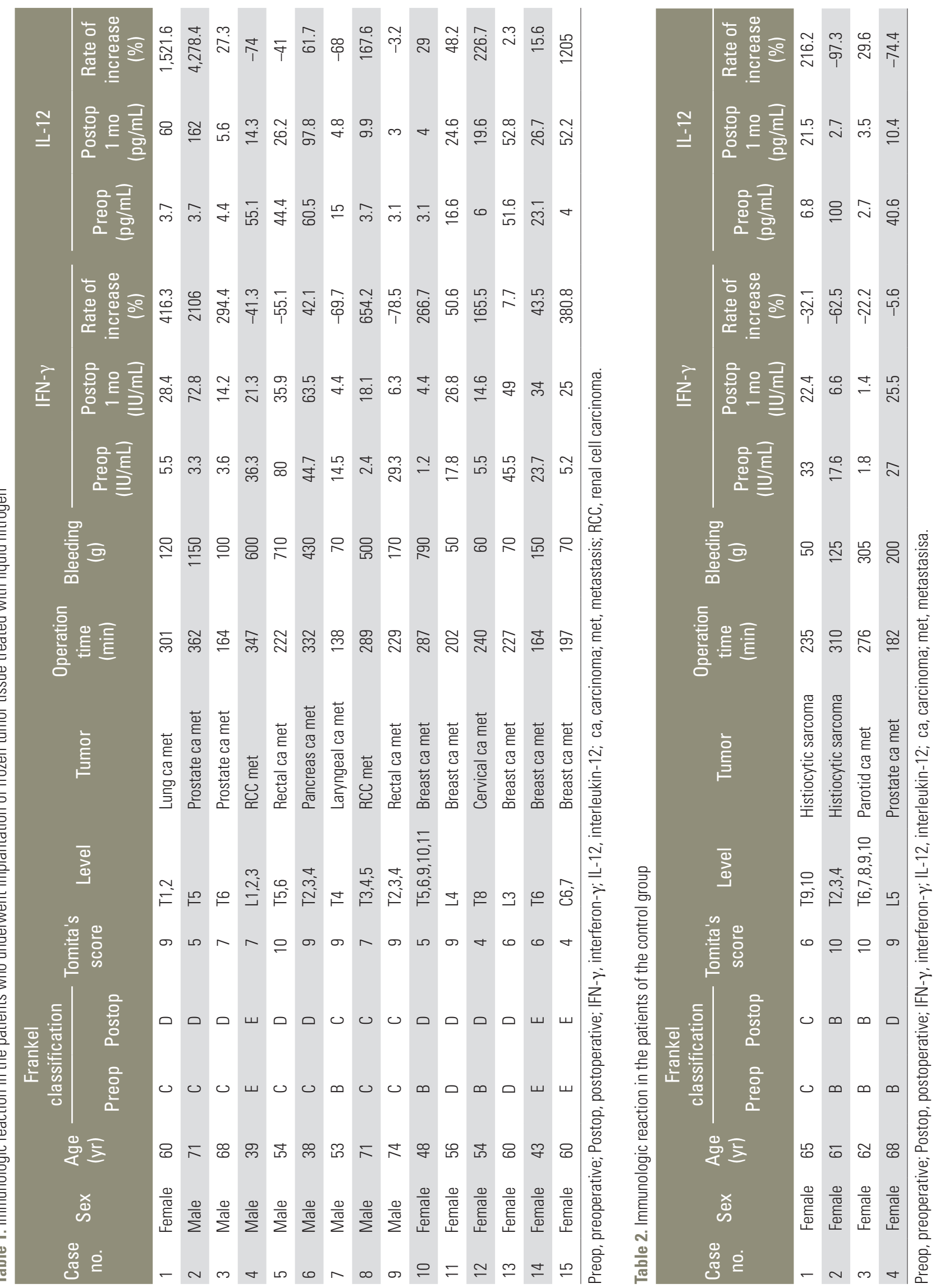
tion group. A portion of the tumor resected during surgery was immersed directly in liquid nitrogen and frozen for 20 minutes. Subsequently, the tumor was taken from liquid nitrogen and thawed at room temperature. At the conclusion of surgery, an approximately $1-\mathrm{cm}$ skin incision was made on one side of the axilla and roughly, $1 \mathrm{~g}$ of the frozen tumor tissue was implanted subcutaneously in the axilla, which was then sutured.

\section{Assessment methods}

Before surgery and at 1 month after surgery, blood test was performed to assess serum interferon $\gamma$ (IFN- $\gamma)$ and interleukin 12 (IL-12) levels. The preoperative levels of IFN- $\gamma$ and IL-12 were compared to their respective levels at 1 month after surgery to calculate their respective rates of increase; also, comparative analysis of rates between the groups was performed. The rates of increase were calculated as follows: (level at 1 month after surgery)-(preoperative level)/(preoperative level) $\times 100$.

\section{Statistical evaluation methods}

The rates of increase in both groups were statistically evaluated using a $t$-test.

\section{Results}

\section{IFN- $\gamma$ Levels}

In the implantation group, the mean IFN- $\gamma$ levels were as follows: before surgery, $21.2 \pm 21.7 \mathrm{IU} / \mathrm{mL}$ and 1 month after surgery, $27.9 \pm 19.9 \mathrm{IU} / \mathrm{mL}$, with a mean rate of increase of $279 \% \pm 530 \%$ (Table 1 ). In the control group, the mean values were $19.9 \pm 11.8$ and $14.0 \pm 10.2 \mathrm{IU} / \mathrm{mL}$, respectively, with a mean rate of increase of $-30.6 \% \pm 20.7 \%$. The mean rate of increase was significantly higher in the implantation group ( $p=0.03$ ) (Fig. 2). Compared with the preoperative levels, the IFN- $\gamma$ levels increased in 11 (73.3\%) of the 15 patients in the implantation group and in none of the 4 patients in the control group.

\section{IL-12 Levels}

In the implantation group, the mean IL-12 levels were as follows: before surgery, $19.9 \pm 20.9 \mathrm{pg} / \mathrm{mL}$ and 1 month after surgery, $37.6 \pm 2.5 \mathrm{pg} / \mathrm{mL}$, with a mean rate of increase

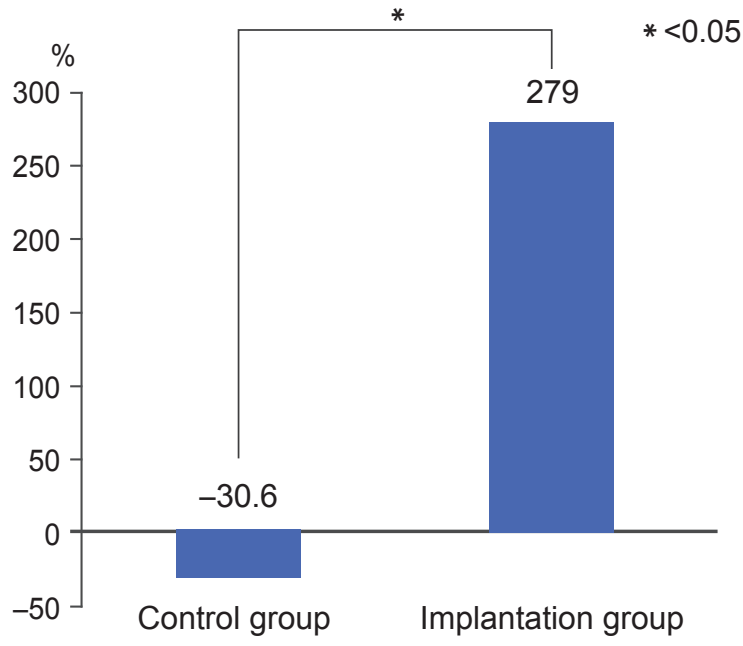

Fig. 2. Rate of increase of the interferon $\gamma$ levels.

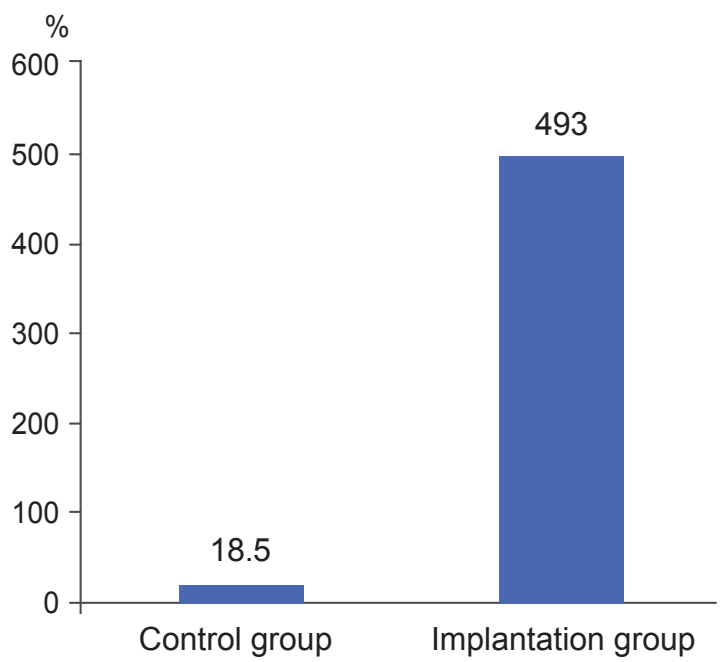

Fig. 3. Rate of increase of the interleukin 12 levels.

of $492 \% \pm 1,111 \%$ (Table 2 ). In the control group, the mean values were $37.5 \pm 39.0$ and $9.5 \pm 7.5 \mathrm{pg} / \mathrm{mL}$, respectively, with a mean rate of increase of $18.5 \% \pm 123 \%$. No significant difference was observed between the groups, although the implantation group exhibited a higher rate of increase ( $p=0.22$ ) (Fig. 3). Compared with the preoperative levels, the IL-12 levels increased in 11 (73.3\%) of the 15 patients in the implantation group and in $2(50 \%)$ of the 4 patients in the control group.

\section{1) Case}

The patient was a 71-year-old man, who was simultaneously diagnosed with prostate cancer and metastatic spinal tumors in the fifth and ninth thoracic vertebrae 2 
years prior to the present investigation. Despite hormone therapy, the metastatic spinal tumors enlarged, resulting in spinal cord compression (Fig. 4). The patient exhibited difficulty in walking due to paraparesis and was referred to our department 4 days later. At the time of admission, the patient presented with Frankel C paraparesis and was unable to stand. Deep tendon reflexes in the legs were enhanced. The metastatic lesions were present at the fifth and ninth thoracic vertebrae; metastasis to other organs was not observed. At the fifth thoracic vertebra, enlargement of the tumor had resulted in spinal cord compression, for which posterior decompression and stabilization were performed. We did not perform surgery on the ninth thoracic vertebra because we did not observe any tumor invasion to the spinal canal (Fig. 5). At the end of surgery, a portion of the resected tumor was frozen by liq-

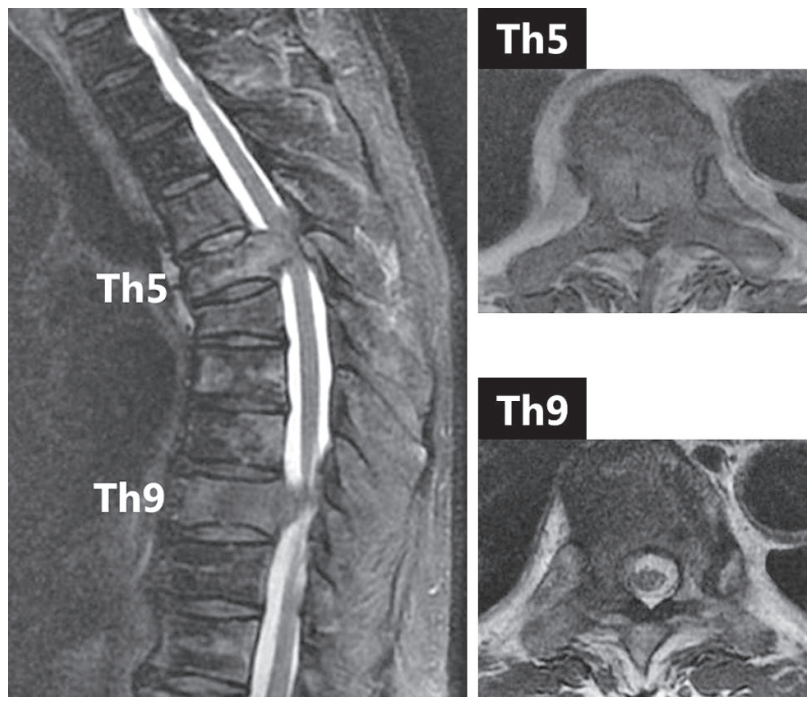

Fig. 4. Preoperative magnetic resonance image. The patient had metastatic invasion to the spinal canal at the T5 level.

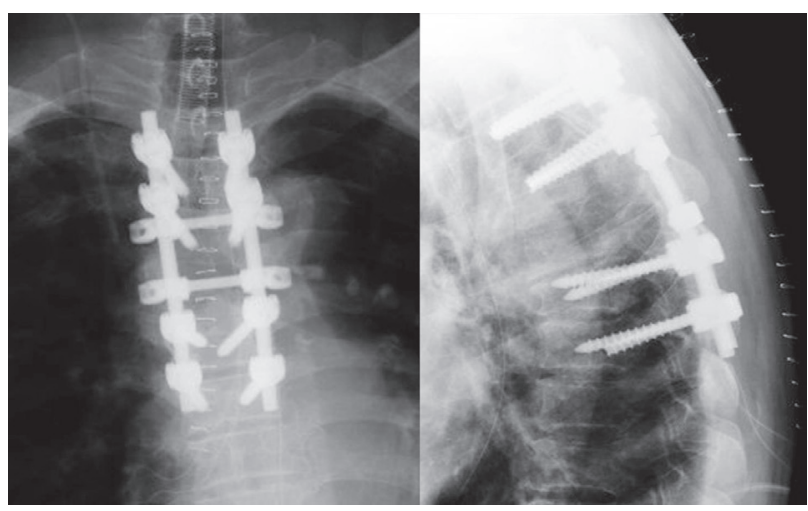

Fig. 5. Postoperative radiographic image. uid nitrogen and implanted subcutaneously in the axilla (Fig. 6). After surgery, the Frankel C paraparesis gradually improved to Frankel D, and the patient regained the ability to walk. The IFN- $\gamma$ and IL-12 levels increased after surgery (from $3.3 \mathrm{IU} / \mathrm{mL}$ before surgery to $72.8 \mathrm{IU} / \mathrm{mL} 1$ month after surgery and from $7.8 \mathrm{pg} / \mathrm{mL}$ before surgery to $162 \mathrm{pg} / \mathrm{mL}$ at 1 month after surgery, respectively). At 1 year after surgery, recurrence of paraparesis due to local recurrence was not seen and enlargement of the metastatic lesion at the ninth thoracic vertebra was not observed.

\section{Discussion}

According to Tomita et al. [3], many patients, in whom posterior decompression and stabilization is suitable have Tomita scores $\geq 6$, which means that a long-term prognosis cannot be anticipated. Furthermore, when invasive surgery is performed in such cases, surgical stress attenuates immunity, which may thus result in rapid exacerbation of other distant metastases. Neeman and Ben-Eliyahu [4] stated that primary tumor resection promotes new metastasis due to seeding micro-carcinoma tissue. In addition, Tai et al. [2] reported that therapy aimed at enhancing Natural killer cell function after tumor extirpation reduces metastatic recurrence and improves the prognoses of cancer patients. The combined use of immunotherapy is reasonable in operations for primary lesions. Additional therapy that further increases their postoperative antitumor immunity is desirable in patients with metastatic spinal tumors, in which tumor cells are disseminated throughout the body.

After confirming through a basic experiment that tumor cells were completely killed by freezing in liquid ni-

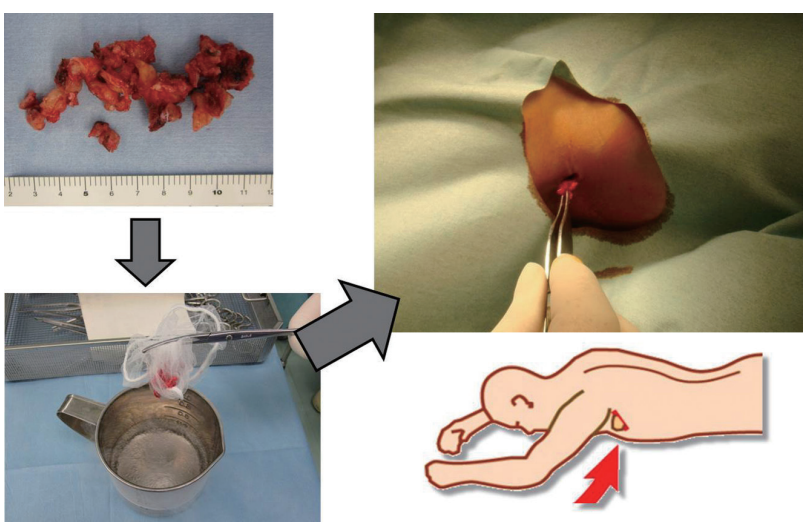

Fig. 6. Implantation of frozen tumor tissue treated with liquid nitrogen. 
trogen for 20 minutes, Tsuchiya et al. [5] obtained favourable outcomes in reconstruction for malignant tumors in the extremities and pelvis using autologous malignant bone treated with liquid nitrogen. In total en bloc spondylectomy, Murakami et al. [6] packed tumor-bearing autograft treated with liquid nitrogen into a cage used for vertebral body reconstruction; in this procedure, no tumor recurrence was observed in the cage. Based on these reports, the risk of local recurrence is considered zero when autologous tumor tissue was implanted after freezing. In fact, in the present study, we observed no local recurrence in the axilla (the implantation site). Nishida et al. [7] used autologous malignant bone treated with liquid nitrogen in reconstruction. Increased IFN- $\gamma$ and IL-12 levels were observed after surgery, when immunity blood analysis was performed before and after surgery, which stated antitumor immunity enhancement effect due to bone treated with liquid nitrogen. Murakami et al. [6] also observed a similar increase in IFN- $\gamma$ and IL-12 levels after total en bloc spondylectomy using bone graft treated with liquid nitrogen. While liquid nitrogen treatment results in complete death of tumor cells, tumor antigens are preserved. Antitumor immunity is considered to be enhanced by the release of tumor antigens from dead tumor cells via dendritic cells [6]. The immune response begins when dendritic cells, which are mainly present in subcutaneous tissue, migrate to lymph nodes. In the present study, we implanted frozen autologous tumor cells in the subcutaneous tissue of the axilla, which is close to the axillary lymph nodes.

Anti-tumor immunity is known to be enhanced by triggering apoptosis in tumor cells in vivo [8,9]. Several studies have used this biological reaction to promote the activation of anti-tumor immunity by subcutaneous administration of dead tumor cells $[10,11]$. Muragaki et al. [10] achieved enhanced antitumor immunity by subcutaneous administration of dead cancer cells using formalin fixation. In addition, Asano et al. [11] achieved enhanced anti-tumor immunity by subcutaneous implantation of dead cancer cells using radiation in mice.

In the present study, we implanted frozen autologous tumor cells and used liquid nitrogen to kill tumor cells, which were administered subcutaneously. As a result, an increase in the IFN- $\gamma$ and IL-12 levels was observed after surgery. Thus, the enhancement of anti-tumor immunity was confirmed. Although we were unable to confirm the reduction of metastatic lesions in the present study, we believe that enhancement of anti-tumor immunity immediately after surgery can circumvent the postoperative attenuation of immunity and thus effectively prolong survival periods. Moreover, the present study was not a randomised controlled trial; thus, randomised controlled trials are desirable in the future. In addition, owing to the small number of patients and the short follow-up period, we were unable to compare survival periods. Although we have confirmed enhancement of immunity in the short term, we were unable to confirm whether this immunity enhancement effect continues over the medium and long terms. Henceforth, in future, further studies are crucial on this issue.

\section{Conclusions}

We demonstrated that decompression and stabilization combined with autologous frozen tumor cell implantation could be performed simultaneously with immunotherapy mediated by dendritic cells. Also, it was confirmed that decompression and stabilization combined with autologous frozen tumor cell implantation could enhance cancer immunity in patients with metastatic spinal tumor. It is hypothesized that this procedure might prevent local recurrence and prolong survival period.

\section{Conflict of Interest}

No potential conflict of interest relevant to this article was reported.

\section{References}

1. Patchell RA, Tibbs PA, Regine WF, et al. Direct decompressive surgical resection in the treatment of spinal cord compression caused by metastatic cancer: a randomised trial. Lancet 2005;366:643-8.

2. Tai LH, de Souza CT, Belanger S, et al. Preventing postoperative metastatic disease by inhibiting surgery-induced dysfunction in natural killer cells. Cancer Res 2013;73:97-107.

3. Tomita K, Kawahara N, Kobayashi T, Yoshida A, Murakami H, Akamaru T. Surgical strategy for spinal metastases. Spine (Phila Pa 1976) 2001;26:298-306.

4. Neeman E, Ben-Eliyahu S. Surgery and stress promote cancer metastasis: new outlooks on perioperative mediating mechanisms and immune involve- 
ment. Brain Behav Immun 2013;30 Suppl:S32-40.

5. Tsuchiya H, Wan SL, Sakayama K, Yamamoto N, Nishida H, Tomita K. Reconstruction using an autograft containing tumour treated by liquid nitrogen. J Bone Joint Surg Br 2005;87:218-25.

6. Murakami H, Demura S, Kato S, et al. Increase of IL12 following reconstruction for total en bloc spondylectomy using frozen autografts treated with liquid nitrogen. PLoS One 2013;8:e64818.

7. Nishida H, Yamamoto N, Tanzawa Y, Tsuchiya H. Cryoimmunology for malignant bone and soft-tissue tumors. Int J Clin Oncol 2011;16:109-17.

8. Clarke C, Smyth MJ. Calreticulin exposure increases cancer immunogenicity. Nat Biotechnol 2007;25:192-3.

9. Obeid M, Tesniere A, Ghiringhelli F, et al. Calreticulin exposure dictates the immunogenicity of cancer cell death. Nat Med 2007;13:54-61.

10. Muragaki Y, Maruyama T, Iseki H, et al. Phase I/IIa trial of autologous formalin-fixed tumor vaccine concomitant with fractionated radiotherapy for newly diagnosed glioblastoma. J Neurosurg 2011;115:24855.

11. Asano K, Nabeyama A, Miyake Y, et al. CD169positive macrophages dominate antitumor immunity by crosspresenting dead cell-associated antigens. Immunity 2011;34:85-95. 